American Journal of Applied Sciences 6 (1): 57-63, 2009

ISSN 1546-9239

(C) 2009 Science Publications

\title{
Response Fusion in Multi-Agent Environment
}

\author{
Fereshteh-Azadi Parand, Nasrollah Moghaddam Charkari and Sara Afrasyabian \\ Tarbiat Modares University, Department of Engineering, Tehran, Iran
}

\begin{abstract}
Multi agent environment are often complex, while each member of agent community has vision about only a part of the environment. In such environment, information fusion of agent community is vital to attain a better accuracy or quality of information. Agents have different ability and can access to information sources with different degree of credibility. As a result, information which is obtained by agents, should be fused with considering their response credibility. In this research, at first we have introduced a method to obtain agent credibility based upon the opinion of agent community. In the proposed method, we measure the possibility of credibility for each agent. Next, we assume that in response to a query, every agent produces a fuzzy answer set in response to a query. On this assumption, a new fuzzy operator with some desired properties for agent responses fusing with their credibility consideration is proposed.
\end{abstract}

Key words: Multi-agent, information fusion, fuzzy operators, possibility theory

\section{INTRODUCTION}

One of the main purpose in agents communication is to achieve the goals better ${ }^{[10]}$. Suppose that in response to a query, each agent can produce a response set which has a level of uncertainty. Uncertainty in agent response may be attributed to two main reasons, namely, deficiency in agent capability and the ambiguity of application information. The first aspect is generally due to agent design/implementation deficiency. The second aspect is due to information overload. For example, in an environment such as the Internet, properties like inaccessibility, nondeterministic and dynamic nature of the information space are sources of agent imprecise decisions. Our goal is the utilization of the other agents in order to reduce the uncertainty and consequently to obtain response with higher quality. For this purpose, the query should propagate in multi-agent environment and agents' response to be aggregate.

For aggregation, these issues should be considered. Agent responses have different degree of accuracy, so final response should be a function of each agent response with considering the degree of credibility. In other words, the opinion of agent with higher credibility has more effect on final answer and vice versa.

For each agent response, the degree of its credibility should be determined. In order to fuse agent responses, we need an operator with considering the problem of credibility degree of resources.
Credibility assignment can be user generated or sanctioned knowledge base ${ }^{[2]}$. In sanction based system a central agent decides about the credibility of the other agents. As there is no centralized element in multiagent environment, sanctioned knowledge base methods might not be used. In our suggested method, the opinion of agents' community is used to assign the credibility to each agent. There are currently some works based on weighting and probability theory for credibility assignment ${ }^{[4,12,13,14,15]}$. Also bayesian ${ }^{[5,6]}$ and fuzzy approach ${ }^{[7,8]}$ are used for credibility assessment. Also in our work the possibility of credibility is assigned to each agent, not the probability of credibility. As the credibility, value assigned by each agent to the other agents is an approximate value and with some uncertainty, assigning credibility possibility is more appropriate than credibility probability.

In the next step, we assume that when an agent receives a query, it propagates the query to the other agents to attain their abilities and opinions. After receiving a query, each agent generates a fuzzy answer set. We need a fusion operator, which considers agent credibility to fuse these answers sets with considering agent credibility. For information fusion by considering source credibility, an operator is suggested by Yager ${ }^{[13]}$ and Prade ${ }^{[9]}$. A problem with this operator applying to fuse agent answers is that the operator on the final decisions does not reflect the low credibility of all agents within the multi-agent environment. Therefore, an environment with low creditable decision makers 
cannot be distinguished from the ones with highly creditable agents. Consequently, a comparison of agent community response is not possible. To resolve this problem, with considering the credibility of the agents, an improved version of this decision fusion operator based upon the assumption that each agent generates a fuzzy decision set is presented.

\section{MATERIALS AND METHODS}

Multi-agent environment is an infrastructure that enables collaborative decision-making. Decision makers may have different degrees of credibility. I

$A=\left\{A_{1}, A_{2}, A_{3}, . ., A_{N}\right\}$. Each of these agents collects information from its accessible knowledge resources and has special capability of decision-making. In this regard, each agent, such as $\mathrm{A}_{\mathrm{i}}$, defines an assigned credibility possibility distribution for each subject.

$$
\text { AsscrePoss }_{\mathrm{i}}=\cup \operatorname{crePosss}_{\mathrm{ij}}
$$

where, crePosss $_{\mathrm{ij}}$ is the degree of credibility possibility assigned by the ith agent to the jth one, while:

$$
\begin{aligned}
& 0 \leq \text { crePoss }_{\text {i1 }} \cup \text { crePoss }_{\mathrm{i} 2} \cup \ldots . . \cup \text { crePoss }_{\text {in }} \leq 1 \\
& \forall \mathrm{DM}_{\mathrm{i}} \in \mathrm{DM}
\end{aligned}
$$

$\mathrm{DP}_{\mathrm{N}}$ is the Nth decision problem, $\mathrm{D}_{\mathrm{k}}$ is the decision which is made for $\mathrm{DP}_{N}$ and $\mathrm{R}_{\mathrm{N}}$ is the membership degree of $D_{k}$ To decision set which is made against $\mathrm{DP}_{\mathrm{N}}$.

The crePosss $\mathrm{i}_{\mathrm{ij}}$ values for each subject are kept in a matrix called the credibility matrix for each subject, as shown in Fig. 1.

\section{CREDIBILITY ASSIGNMENT TO AGENTS}

After the matrix CrePoss is established, the credibility possibility of each agent crePosij, should be influenced by the opinions of the other agents.

Credibility assignment to each agent is defined by a fuzzy relation implemented as a max-min composition. At each instance of time, $\mathrm{t}+1$, the maxmin composition influences the opinion of each agent, $\operatorname{crePoss}_{\mathrm{ij}}^{\mathrm{t}+1}$ by the others opinions as follows: Suppose there is a group of $\mathrm{N}$ agents in multi-agent environment, indexed by the set

$$
\begin{aligned}
\operatorname{crePoss}_{\mathrm{ij}}^{\mathrm{t}+1} & =\max \left\{\operatorname{crePoss}_{\mathrm{ik}} \wedge \operatorname{crePoss}_{\mathrm{kj}}^{\mathrm{t}}\right\} \mathrm{k}=1, \ldots, \mathrm{N} \\
\text { crePoss }_{\mathrm{ij}}^{1} & =\operatorname{crePoss}_{\mathrm{ij}}
\end{aligned}
$$

$$
\left[\begin{array}{llll}
\operatorname{crePoss}_{11}\left(\text { Subject }_{\mathrm{m}}\right) & \operatorname{crePoss}_{12}\left(\text { Subject }_{\mathrm{m}}\right) & \ldots & \operatorname{crePoss}_{\mathrm{in}}\left(\text { Subject }_{\mathrm{m}}\right) \\
\operatorname{crePoss}_{21}\left(\text { Subject }_{\mathrm{m}}\right) & \operatorname{crePoss}_{22}\left(\operatorname{Subject}_{\mathrm{m}}\right) & \ldots & \operatorname{crePoss}_{2 \mathrm{n}}\left(\text { Subject }_{\mathrm{m}}\right) \\
& & & \\
\operatorname{crePoss}_{\mathrm{N} 1}\left(\text { Subject }_{\mathrm{m}}\right) & \operatorname{crePoss}_{\mathrm{nn}}\left(\operatorname{Subject}_{\mathrm{m}}\right)
\end{array}\right]
$$

Fig. 1: Credibility possibility matrix

Our goal is to gain a possibility distribution for the credibility of the agents by a distributed model. In our model, crePosss $\mathrm{s}_{\mathrm{ij}}$, the credibility of the agent $\mathrm{Ai}$ is dependent on, the amount of the credibility that all agents $\mathrm{DM}_{\mathrm{k}}$ for $\mathrm{k}=1$ to $\mathrm{N}$, have assigned to the ith agent. The credibility of an agent, crePosss $s_{\mathrm{ij}}$, is a function of $\operatorname{crePosss}_{\mathrm{ij}} \mathrm{k}=1, \ldots, \mathrm{N}$.

$$
\operatorname{crePoss}_{\mathrm{i}}=\overline{\mathrm{F}}\left(\operatorname{crePoss}_{\mathrm{ki}}, \operatorname{crePoss}_{\mathrm{k}}\right), \mathrm{k}=1 . . \mathrm{N}
$$

Here, $\overline{\mathrm{F}}$ indicates the max-min function. To apply the max-min function, a set of equations $\overrightarrow{\text { crePoss }}=\overrightarrow{\text { crePoss }}$ o $\mathrm{P}^{\mathrm{n}}$ is established where, vector $\overrightarrow{\text { crePoss }}=\left\langle\operatorname{crePoss}_{1}, \operatorname{crePoss}_{2}, \ldots\right.$, crePoss $\left._{N}\right\rangle$ indicates the credibility possibility distribution for the agents community; $\mathrm{o}$ is the max-min operator and $\mathrm{P}$ is a matrix whose components are the degree of credibility of each agent from the point of view of the other agents.

Theorem 1: If an agent $A_{i}$ increases crePoss $s_{i k}$, then from the point of view of $\mathrm{Ai}$ the $\mathrm{A}_{\mathrm{k}}$ 's capability in determining the credibility of the other agents will not be reduced.

Proof: Let the credibility value of the kth agent from the point of view of the ith agent, crePosss $_{\mathrm{ik}}$, changes from $\alpha$ to $\beta$ such that $\beta \succ \alpha$. In this case, $\alpha \wedge \operatorname{crePoss}_{\mathrm{kj}}^{\mathrm{t}} \leq \beta \wedge \operatorname{crePoss}_{\mathrm{kj}}^{\mathrm{t}}$, where j represents any agent in the multi-agent environment. As a result crePoss $_{i k} \wedge \operatorname{crePoss}_{\mathrm{kj}}^{\mathrm{t}}$ will not decrease and the possibility that $\beta \wedge \operatorname{crePoss}_{\mathrm{kj}}^{\mathrm{t}}$ be the maximum value of $\left\{\right.$ crePoss $_{i p} \wedge$ crePoss $\left._{p j}^{t}\right\} \quad P=1, \ldots, N$ will increase. Since $\operatorname{crePoss}_{\mathrm{kj}}^{\mathrm{t}}$ indicates the credibility degree of $\mathrm{Aj}$ in judgment of $\mathrm{Ak}$ at time $\mathrm{t}$, the role of $\mathrm{Ak}$ in determination of the credibility of $\mathrm{Aj}$ will not decrease.

Theorem 2: If crePoss o AssCreM $=$ crePoss Then: crePoss o AssCreM $^{\mathrm{n}}=$ crePoss $^{[2]}$.

Considering theorem 2, to work out the value of the credibility vector, crePoss, instead of using the relation crePossoP $^{\mathrm{n}}=$ crePoss, the relation 
crePoss o AssCreM $=$ crePoss can be used to obtain a distribution, crePoss. Such a distribution is called stationary distribution.

Example 1: Suppose there are three agents $A=\{A 1$, $\mathrm{A} 2, \mathrm{~A} 3 \mathrm{\}}$, a max function, $\mathrm{f}$ and a min function, $\mathrm{g}$. It is desirable to calculate the credibility possibility of the second agent such that the condition of the function $\mathrm{F}$, defined in relation (2), is satisfied.

Using theorem 2 and Eq. 2, the possibility measure of the second agent credibility computes as follows:

$$
\begin{aligned}
& \operatorname{crePoss}_{2}=\mathrm{f}\left(\mathrm{g}\left(\mathrm{crePoss}_{1}, \mathrm{crePoss}_{12}\right),\right. \\
& \left.\mathrm{g}\left(\mathrm{crePoss}_{2}, \operatorname{poss}_{22}\right), \mathrm{g}\left(\mathrm{crePoss}_{3}, \operatorname{poss}_{32}\right)\right)
\end{aligned}
$$

where, crePoss $_{1}, \mathrm{crePoss}_{2}$ and crePoss $_{3}$ represent the credibility of the first, second and third agents respectively and crePoss is the credibility matrix.

\section{DECISION FUSION}

As described above, for a collection of $\mathrm{N}$ agents, indexed by the set $\mathrm{A}=\{\mathrm{A} 1, \mathrm{~A} 2, \ldots, \mathrm{AN}\}$, the credibility possibility could be estimated for each of the agents, based upon the other agents opinions. The distribution of the estimated credibility possibility values is represented as a vector crePoss $=\left\langle\operatorname{crePoss}_{1}, \operatorname{crePoss}_{2}, \ldots \ldots, \operatorname{crePoss}_{\mathrm{N}}\right\rangle, \quad$ where crePoss $_{i}$ is the credibility of $\mathrm{Ai}$. In this research, the vector $\overrightarrow{\text { crePoss }}$ is applied to fuse decisions made by the members of agent community. In response to any decision making problem such as $\mathrm{q}$, Ai generates a fuzzy decision set $\overline{R_{i}}(D P)=\{D 1, D 2, \ldots, D m\}$, with the perception of its accessible knowledge environment and its belief, such that each fuzzy member $D_{k} \in \overline{R_{i}}(D P)$ is one of the $\mathrm{m}$ decisions made by DMi. The number of selected decisions, m, may vary for different agents. The membership degree of $D_{k}$ to the final set of decisions or responses $\overline{R_{F}}(D P)$ is represented as $\overline{R_{F}}\left(D P, D_{k}\right)$ such that $0 \leq \overline{R_{F}}\left(D P, D_{k}\right) \leq 1$. The goal is to estimate $\overline{\mathrm{R}_{\mathrm{F}}}\left(\mathrm{DP}, \mathrm{D}_{\mathrm{k}}\right)$ based upon the possibility distribution of agents' credibility, crePoss, and the membership degree of $D_{k}$ to the decision set, $D$, of each agent. Estimating the membership degree, $\overline{R_{i}}\left(q, D_{k}\right)$, is formalized as follows:

$$
\begin{aligned}
& \mathrm{R}_{\mathrm{F}}\left(\mathrm{DP}, \mathrm{D}_{\mathrm{k}}\right)=\mathrm{f}\left(\text { crePoss }_{\mathrm{i}}, \mathrm{R}_{\mathrm{i}}\left(\mathrm{DP}, \mathrm{D}_{\mathrm{k}}\right)\right) \\
& 0 \leq \mathrm{R}_{\mathrm{F}}\left(\mathrm{DP}, \mathrm{D}_{\mathrm{k}}\right) \leq 1 \quad \forall \mathrm{A}_{\mathrm{i}} \in \mathrm{A}
\end{aligned}
$$

We choose function $\mathrm{F}$ such that it has the following eight properties which are required for any information fusion operator $^{[14]}$.

Property 1: Monotonicity with respect to the membership degree of each decision to decision set:

Let

$$
\begin{gathered}
\mathrm{R}_{\mathrm{F}}\left(\mathrm{DP}, \mathrm{D}_{\mathrm{k}}\right)=\mathrm{f}\left(\text { crePoss }_{\mathrm{i}}, \mathrm{R}_{\mathrm{i}}\left(\mathrm{DP}, \mathrm{D}_{\mathrm{k}}\right)\right), \\
\forall \mathrm{A}_{\mathrm{i}} \in \mathrm{A}, \quad \mathrm{i}=1, \ldots, \mathrm{N}
\end{gathered},
$$

$\mathrm{R}_{\mathrm{F}}\left(\mathrm{DP}, \mathrm{D}_{1}\right)=\mathrm{f}\left(\mathrm{crePoss}_{\mathrm{i}}, \mathrm{R}_{\mathrm{i}}\left(\mathrm{DP}, \mathrm{D}_{1}\right)\right) \forall \mathrm{A}_{\mathrm{i}} \in \mathrm{A} \quad$ and

$\mathrm{R}_{\mathrm{i}}\left(\mathrm{DP}, \mathrm{D}_{\mathrm{k}}\right) \geq \mathrm{R}_{\mathrm{i}}\left(\mathrm{DP}, \mathrm{D}_{1}\right) \forall \mathrm{A}_{\mathrm{i}} \in \mathrm{A}$

Then

$\mathrm{R}_{\mathrm{F}}\left(\mathrm{DP}, \mathrm{D}_{\mathrm{k}}\right) \geq \mathrm{R}_{\mathrm{F}}\left(\mathrm{DP}, \mathrm{D}_{1}\right)$.

It implies that if all the members of agent community, DM, make two decisions $\mathrm{D}_{\mathrm{k}}$ and Dl then based on the assumption that all agents agreement on Dk being either identical to or more acceptable than D1, it can be concluded that Dk has greater membership degree to final decision set than D1 has.

Property 2: Monotonicity with respect to credibility possibility:

$$
\begin{aligned}
& \text { If } \quad \mathrm{R}_{\mathrm{F}}^{\prime}\left(\mathrm{DP}, \mathrm{D}_{1}\right)=\mathrm{f}\left(\operatorname{crePoss}_{\mathrm{i}}^{\prime}, \mathrm{R}_{\mathrm{i}}^{\prime}\left(\mathrm{DP}, \mathrm{D}_{1}\right)\right) \\
& \forall \mathrm{DM}_{\mathrm{i}}^{\prime} \in \mathrm{DM}^{\prime} \\
& \mathrm{R}_{\mathrm{F}}\left(\mathrm{DP}, \mathrm{D}_{\mathrm{k}}\right)=\mathrm{f}\left(\mathrm{crePoss}_{\mathrm{i}}, \mathrm{R}_{\mathrm{i}}\left(\mathrm{DP}, \mathrm{D}_{\mathrm{k}}\right)\right) \forall \mathrm{DM}_{\mathrm{i}} \in \mathrm{DM} \quad \text { and } \\
& \mathrm{R}_{\mathrm{i}}\left(\mathrm{DP}, \mathrm{D}_{\mathrm{k}}\right)=\mathrm{R}_{\mathrm{i}}^{\prime}\left(\mathrm{DP}, \mathrm{D}_{\mathrm{l}}\right), \operatorname{crePoss}_{\mathrm{i}} \geq \text { crePoss }_{\mathrm{i}}^{\prime} \text { then } \\
& \mathrm{R}_{\mathrm{F}}^{\prime}\left(\mathrm{DP}, \mathrm{D}_{1}\right) \geq \mathrm{R}_{\mathrm{F}}\left(\mathrm{DP}, \mathrm{D}_{\mathrm{k}}\right) \text {. }
\end{aligned}
$$

Assume Dl and Dk are two decisions made by $\mathrm{DM}_{\mathrm{i}}$ and $\mathrm{DM}_{\mathrm{i}}^{\prime}$ in response to a DP respectively while $\mathrm{R}_{\mathrm{i}}^{\prime}\left(\mathrm{DP}, \mathrm{D}_{1}\right) \quad$ and crePoss $_{\mathrm{i}} \geq$ crePoss $_{\mathrm{i}}^{\prime}$ $\forall \mathrm{DM}_{\mathrm{i}} \in \mathrm{DM}, \mathrm{DM}_{\mathrm{i}}^{\prime} \in \mathrm{DM}^{\prime}$. In this regard, the membership degree $D_{1}$ to final decision set, $R_{F}^{\prime}\left(D P, D_{1}\right)$, will be more than or equal to the membership degree of $D_{k}$ to final Decision set, $R_{F}\left(D P, D_{k}\right)$. This property is applicable when two or more knowledge multi-agent are compared.

Property 3: Pointwiseness property: The determination of $R_{F}\left(D P, D_{k}\right)$ value depends only on $\mathrm{R}_{\mathrm{i}}\left(\mathrm{DP}, \mathrm{D}_{\mathrm{k}}\right)$ and crePoss $\mathrm{i}_{\mathrm{i}} \forall \mathrm{DM}_{\mathrm{i}} \in \mathrm{DM}$ and is independent of $\mathrm{R}_{\mathrm{i}}\left(\mathrm{DP}, \mathrm{D}_{1}\right) \forall \mathrm{DM}_{\mathrm{i}} \in \mathrm{DM}, 1 \neq \mathrm{k}$.

Property 4: If the possibility of credibility of jth agent is more than the possibility of credibility of the kth agent, crePoss ${ }_{j}>\operatorname{crePos}_{k}$, then the ability of jth agent in 
determination of final decision set should be equal to or more than the ability of kth agent in determination of final decision set. In other words, the less credible agent should have fewer roles in determination of final decision set and vice versa.

Property 5: If credibility possibility of an agent is zero, then its opinion has no effect on the result of function $\mathrm{f}$.

Property 6: If the credibility possibility of an agent is one, then its decision impact should not be reduced and its constraints on the answer should be imposed completely.

Property 7: Commutatively property: Indexing of agents should not affect the final decision set. For instance, if our multi-agent environment has two agents indexed one and two then:

$\mathrm{f}\left(\left(\operatorname{crePoss}_{1}, \mathrm{R}_{1}\left(\mathrm{DP}, \mathrm{D}_{\mathrm{k}}\right)\right),\left(\operatorname{crePoss}_{2}, \mathrm{R}_{2}\left(\mathrm{DP}, \mathrm{D}_{\mathrm{k}}\right)\right)\right)$

$=\mathrm{f}\left(\left(\operatorname{crePoss}_{2}, \mathrm{R}_{2}\left(\mathrm{DP}, \mathrm{D}_{\mathrm{k}}\right)\right),\left(\operatorname{crePoss}_{1}, \mathrm{R}_{1}\left(\mathrm{DP}, \mathrm{D}_{\mathrm{k}}\right)\right)\right)$

Property 8: $\mathrm{R}_{\mathrm{F}}\left(\mathrm{DP}, \mathrm{D}_{1}\right)<\max \left(\operatorname{crePoss}\left(\mathrm{DM}_{\mathrm{i}}\right)\right) \forall \mathrm{D}_{1} \in \mathrm{D}$

A community of agents in multi-agent environment can not produce a decision which its membership degree to the final decision set is greater than the credibility degree of most credible agent.

For example if the $\max \left(\right.$ crePoss $\left._{\mathrm{i}}\right)=0.8$ then $\max \left(\mathrm{R}_{\mathrm{F}}\left(\mathrm{DP}, \mathrm{D}_{1}\right)\right) \leq 0.8 \quad \forall \mathrm{A}_{\mathrm{i}} \in \mathrm{A}, \forall \mathrm{D}_{1} \in \mathrm{D}$

\section{SOLUTION OF THE EQUATION SET}

In order to solve the equation set crePoss o crePossM = crePoss, the fuzzy markov chain mode $^{[1]}$ is used. Since crePossM is a fuzzy transitive matrix, crePoss should be an eigen fuzzy set. Also, since crePoss is a possibility distribution, it is appropriate to obtain the greatest eigen fuzzy set satisfying the equation set crePoss o crePossM = crePoss .

Example 2: Suppose that the credibility matrix crePossM is as shown Fig. 2. It is desired to obtain he credibility vector, crePoss.

$$
\text { crePossM }=\left[\begin{array}{ccccc}
0.1 & 0.7 & 0.2 & 0.8 & 0.7 \\
0 & 0.6 & 0.4 & 0.3 & 0.5 \\
0.3 & 1 & 0 & 01 & 0.4 \\
0.3 & 0.3 & 0.8 & 0.1 & 0 \\
0 & 0 & 0.7 & 0.5 & 0
\end{array}\right]
$$

Fig. 2: CrePoss matrix for example 2
For obtaining the greatest eigen fuzzy set we use the algorithm which presented in ${ }^{[15]}$.

If we have xo $\mathrm{P}=\mathrm{P}$ equation the stages of algorithm are as follows:

- Determine first x1 with the elements corresponding to the greatest elemen tcolumn of $\mathrm{P}$

- Compute $\mathrm{P} 2=\mathrm{Po} \mathrm{P}$ and determine the greatest elements in each column of $\mathrm{P} 2$ they give $\mathrm{x} 2$

- Compare x2 with $\mathrm{x} 1$ : if they are different, compute $\mathrm{P} 3=\mathrm{P}$ o $\mathrm{P} 2$ to get $\mathrm{x} 3$

- Compare x3 with x 1: if they are different, compute $\mathrm{P} 4=\mathrm{Po}$ P3 to get $\mathrm{x} 4$, stop

It is found $\mathrm{m}$ such that $\mathrm{xm}+1=\mathrm{xm}$, that is $\mathrm{xm}=\mathrm{xmo} \mathrm{P}$. Applying this algorithm, the greatest eigen fuzzy set, or in the other words the possibility distribution vector, crePoss and the possibility of crePoss will be as follows:

$$
\operatorname{CrePoss}_{\mathrm{x}}=\left[\begin{array}{lllll}
0.3 & 0.6 & 0.5 & 0.5 & 0.5
\end{array}\right]
$$

possibilly $\left(\right.$ crePoss $\left._{\mathrm{x}}\right)=\max (0.3,0.6,0.5,0.5,0.5)=0.6$

So far the possibilities of credibility for each of agents according to the other agents' opinions is evaluated. In the next step, the evaluated credibility values are used to fuse the fuzzy decision set of the members of agents' community.

\section{SUGGESTED OPERATOR FOR DECISION FUSION}

In this research, to evaluate RF function, an information fusion operator considering the properties discussed in decision fusion section is presented. Some works on information fusion operator based on source credibilty have been done by Dubios and Prade ${ }^{[3]}$.

According to relation 2, This operator modifies rank of answers and then selects the minimum of these modified values. The drawback of this operator is that it has not monotonic properties with respect to credibility possibility. Therefore, it could not be used for comparing different multi-agent environment or aggregating of decision sets of more than one multiagent environment. In order to use the suggested operator, considering the degree of credibility possibility of each agent, $\overline{R_{i}}\left(D P, D_{k}\right)$ is modified to $\overline{\mathrm{R}_{\mathrm{i}}^{\prime}}\left(\mathrm{DP}, \mathrm{D}_{\mathrm{k}}\right)$.

$$
\mathrm{R}_{\mathrm{i}}^{\prime}\left(\mathrm{DP}, \mathrm{D}_{\mathrm{k}}\right)=\mathrm{R}_{\mathrm{i}}\left(\mathrm{DP}, \mathrm{D}_{\mathrm{k}}\right) \vee\left(1-\operatorname{crePoss}_{\mathrm{i}}\right)
$$

Then we use of $\overline{R_{i}^{\prime}}\left(D P, D_{k}\right)$ to gain $R_{F}\left(D P, D_{K}\right)$ in the following way: 
Am. J. Applied Sci., 6 (1): 57-63, 2009

Table 1: Response of agents to each query

\begin{tabular}{|c|c|c|c|c|c|}
\hline D5 & D4 & D3 & D2 & D1 & \\
\hline N/A & N/A & 0.4 & 0.6 & 0.8 & R1 \\
\hline 0.8 & N/A & N/A & 1 & 0.6 & R2 \\
\hline 1 & 0.3 & 0.9 & 0.7 & 0.4 & $\mathrm{R} 3$ \\
\hline N/A & 0.8 & 0.6 & 0.8 & 0.5 & R4 \\
\hline N/A & 0.1 & 0.3 & 0.7 & N/A & R5 \\
\hline
\end{tabular}

Table 2: Modified Response considering credibility

\begin{tabular}{llllll}
\hline $\mathrm{D} 5$ & $\mathrm{D} 4$ & $\mathrm{D} 3$ & $\mathrm{D} 2$ & $\mathrm{D} 1$ & \\
\hline 0.7 & 0.7 & 0.7 & 0.7 & 0.8 & $\mathrm{R}_{1}^{\prime}$ \\
0.8 & 0.4 & 0.4 & 1 & 0.6 & $\mathrm{R}_{2}^{\prime}$ \\
1 & 0.5 & 0.9 & 0.7 & 0.5 & $\mathrm{R}_{3}^{\prime}$ \\
0.5 & 0.8 & 0.6 & 0.8 & 0.5 & $\mathrm{R}_{4}^{\prime}$ \\
0.5 & 0.5 & 0.5 & 0.7 & 0.5 & $\mathrm{R}_{5}^{\prime}$ \\
\hline
\end{tabular}

$$
\begin{gathered}
\mathrm{R}_{\mathrm{F}}\left(\mathrm{DP}, \mathrm{D}_{\mathrm{k}}\right)=\min \left(\mathrm{R}_{\mathrm{i}}^{\prime}\left(\mathrm{DP}, \mathrm{D}_{\mathrm{k}}\right)\right) \wedge \max \left(\mathrm{crePoss}_{\mathrm{i}}\right) \\
\mathrm{i}=1,2, \ldots, \mathrm{N}
\end{gathered}
$$

Example 3: Considering the possibility distribution of example 2, crePoss $_{\mathrm{x}}$, as credibility values and Table 1 as decision sets for agents' community then the modified memberships are the values presented in Table 2. The membership degree of D1 is obtained in the following way.

\section{PROOF THE CORRECTNESS OF THE OPERATOR}

In this research, it is proved that the new decision fusion operator, $\mathrm{R}_{\mathrm{f}}$, introduced in previous section, satisfies properties number 1 to 8 discussed in decision fusion section.

Property 1: If all agents DMi believe that the rank, Ri, of decision Dk for the problem DP is greater than rank Dl then considering the credibility possibility of the agents, the modified rank, $R_{i}^{\prime}$, of decision $D k$ is greater than $\mathrm{R}_{\mathrm{i}}^{\prime}(\mathrm{DP}, 1)$. In the other words;

$$
\text { If } \mathrm{R}_{\mathrm{i}}\left(\mathrm{DP}, \mathrm{D}_{\mathrm{k}}\right) \geq \mathrm{R}_{\mathrm{i}}\left(\mathrm{DP}, \mathrm{D}_{1}\right) \forall \mathrm{DM}_{\mathrm{i}} \in \mathrm{DM}
$$
then considering T-conorm (here $v)$ -
$R_{i}^{\prime}\left(D P, D_{k}\right) \geq R_{i}^{\prime}\left(D P, D_{1}\right)$.

Therefore;

If $R_{p}^{\prime}\left(D P, D_{k}\right)$ is the smallest element in the set $\mathrm{R}_{\mathrm{i}}^{\prime}\left(\mathrm{q}, \mathrm{D}_{\mathrm{k}}\right) \forall \mathrm{DM}_{\mathrm{i}} \in \mathrm{DM}$ then there is an element less than or equal to $R_{p}^{\prime}\left(D P, D_{k}\right)$ in the set $\mathrm{R}_{\mathrm{i}}^{\prime}\left(\mathrm{DP}, \mathrm{D}_{1}\right) \quad \forall \mathrm{DM}_{\mathrm{i}} \in \mathrm{DM}$. In the other words: $\min \left(\mathrm{R}_{\mathrm{i}}^{\prime}\left(\mathrm{DP}, \mathrm{D}_{\mathrm{k}}\right)\right) \geq \min \left(\mathrm{R}_{\mathrm{i}}^{\prime}\left(\mathrm{DP}, \mathrm{D}_{1}\right)\right) \forall \mathrm{DM}_{\mathrm{i}} \in \mathrm{DM}$

Considering the property of T-norm operators, we have:

$$
\begin{aligned}
& \min \left(\mathrm{R}_{\mathrm{i}}^{\prime}\left(\mathrm{DP}, \mathrm{D}_{\mathrm{k}}\right)\right) \wedge \max \left(\text { crePoss }_{\mathrm{i}}\right) \geq \\
& \min \left(\mathrm{R}_{\mathrm{i}}^{\prime}\left(\mathrm{DP}, \mathrm{D}_{1}\right)\right) \wedge \max \left(\text { crePoss }_{\mathrm{i}}\right) \forall \mathrm{DM}_{\mathrm{i}} \in \mathrm{DM}
\end{aligned}
$$

Consequently $\mathrm{R}_{\mathrm{F}}\left(\mathrm{DP}, \mathrm{D}_{\mathrm{k}}\right) \geq \mathrm{R}_{\mathrm{F}}\left(\mathrm{DP}, \mathrm{D}_{1}\right)$.

Therefore, the introduced operator satisfies property one.

Property 2: If there are two collection of agents D and D* such that:

$$
\left(\operatorname{crePoss}_{\mathrm{i}^{*}}\right) \geq\left(\operatorname{crePoss}_{\mathrm{i}}\right) \forall \mathrm{D}_{\mathrm{i}} \in \mathrm{D}, \forall \mathrm{D}_{\mathrm{i}^{*}} \in \mathrm{D}^{*}
$$

Then $\max \left(\right.$ crePoss $\left._{\mathrm{i}^{*}}\right) \geq \max \left(\operatorname{crePoss}_{\mathrm{i}}\right)$.

In addition, it is obvious that:

$$
\mathrm{R}_{\mathrm{F}}\left(\mathrm{DP}, \mathrm{D}_{\mathrm{k}}\right) \leq \max \left(\mathrm{crePoss}_{\mathrm{i}}\right)
$$

and

$$
\mathrm{R}_{\mathrm{F}}^{*}\left(\mathrm{DP}, \mathrm{D}_{1}\right) \leq \max \left(\operatorname{crePoss}_{\mathrm{i}^{*}}\right)
$$

As a result $\mathrm{R}_{\mathrm{F}}\left(\mathrm{DP}, \mathrm{D}_{\mathrm{k}}\right) \leq \mathrm{R}_{\mathrm{F}}{ }^{*}\left(\mathrm{DP}, \mathrm{D}_{1}\right)$.

Therefore, it is observed that the introduced operator, RF, satisfies property two.

Property 3: RF satisfies pointwiseness property as $\mathrm{R}_{\mathrm{i}}\left(\mathrm{DP}, \mathrm{D}_{\mathrm{k}}\right) \forall \mathrm{D}_{\mathrm{i}} \in \mathrm{D}$ and crePoss $_{\mathrm{i}}$ are only used to calculate $R_{F}\left(D P, D_{k}\right)$.

Property 4: The higher the credibility possibility of agent, the higher its effect on the final response or decision RF. According to relation (3), $R_{F}\left(D P, D_{1}\right)$ is a conjunction of two parameters. The first parameter is $\max \left(\operatorname{crePoss}_{\mathrm{i}}\right) \forall \mathrm{DM}_{\mathrm{i}} \in \mathrm{DM}$ and the second is $\min \mathrm{R}_{\mathrm{i}}^{\prime}\left(\mathrm{DP}, \mathrm{D}_{1}\right) \forall \mathrm{DM}_{\mathrm{i}} \in \mathrm{DM}$. Considering the first parameter, it is observed that only the highest credible agent DMi determines the value of the first parameter.

Considering the second parameter which is a conjunction of $\left(1-\right.$ crePoss $\left._{\mathrm{i}}\right)$ and $\mathrm{R}_{\mathrm{i}}\left(\mathrm{DP}, \mathrm{D}_{1}\right)$, it is observed that if:

$$
\text { crePossj } \geq \text { crePossk }
$$

and 
1 -crePossj $\leq 1$-crePossk

then according to the T-conorm property the following relations are always true:

$$
\begin{aligned}
& \mathrm{R}_{\mathrm{j}}^{\prime}>1-\operatorname{crePoss}_{\mathrm{j}} \\
& \mathrm{R}_{\mathrm{k}}^{\prime}>1-\operatorname{crePoss}_{\mathrm{k}}
\end{aligned}
$$

it can be concluded that $R_{j}^{\prime} \geq R_{k}^{\prime}$. As a result $R_{j}^{\prime}$ could not be the min $\left(\mathrm{R}_{\mathrm{i}}^{\prime}\right) \quad \forall \mathrm{DM} \mathrm{M}_{\mathrm{i}} \in \mathrm{DM}$, so it has no effect in determination of RF.

So the introduced operator satisfies the forth property.

Property 5: If the credibility possibility of the ith agent is zero, then according to the relation (2), the ith agent has no effect on the value of RF, because:

$$
\begin{aligned}
& \text { crePoss }_{j}=0 \Rightarrow 1-\text { crePoss }_{j}=1 \Rightarrow \\
& \mathrm{R}_{\mathrm{j}}^{\prime}\left(\mathrm{DP}, \mathrm{D}_{\mathrm{k}}\right)=1 \quad \forall \mathrm{D}_{\mathrm{k}} \in \mathrm{D}
\end{aligned}
$$

Moreover, since:

$$
\begin{aligned}
& \mathrm{R}_{\mathrm{F}}\left(\mathrm{DP}, \mathrm{D}_{\mathrm{k}}\right)=\min \left(\mathrm{R}_{1}^{\prime}\left(\mathrm{DP}, \mathrm{D}_{\mathrm{k}}\right), \mathrm{R}_{\mathrm{j}}^{\prime}\left(\mathrm{DP}, \mathrm{D}_{\mathrm{k}}\right)\right) \\
& \left.\wedge \max \left(\mathrm{crePoss}_{1}, \operatorname{crePoss}_{\mathrm{j}}\right)\right) \\
& \quad \forall \mathrm{DM}_{1} \in \mathrm{DM}-\left\{\mathrm{DM}_{\mathrm{j}}\right\} \\
& \left.\mathrm{R}_{\mathrm{F}}\left(\mathrm{DP}, \mathrm{D}_{\mathrm{k}}\right)=\min \left(\mathrm{R}_{1}^{\prime}\left(\mathrm{DP}, \mathrm{D}_{\mathrm{k}}\right), 1\right) \wedge \max \left(\operatorname{crePoss}_{1}, 0\right)\right) \\
& \quad \forall \mathrm{DM}_{1} \in \mathrm{DM}-\left\{\mathrm{DM}_{\mathrm{j}}\right\} \\
& \left.\mathrm{R}_{\mathrm{F}}\left(\mathrm{DP}, \mathrm{D}_{\mathrm{k}}\right)=\min \left(\mathrm{R}_{1}^{\prime}\left(\mathrm{DP}, \mathrm{D}_{\mathrm{k}}\right)\right) \wedge \max \left(\operatorname{crePoss}_{1}\right)\right) . \\
& \quad \forall \mathrm{DM}_{1} \in \mathrm{DM}-\left\{\mathrm{DM}_{\mathrm{j}}\right\}
\end{aligned}
$$

Property 6: If the credibility possibility of the ith agent is one, then according to the relation (2), the ith decision-maker opinion will be imposed completely as it is. In the other words:

$$
\begin{aligned}
& \operatorname{crePoss}_{\mathrm{j}}=1 \Rightarrow 1-\text { crePoss }_{\mathrm{j}}=0 \Rightarrow \\
& \mathrm{R}_{\mathrm{j}}^{\prime}\left(\mathrm{DP}, \mathrm{D}_{\mathrm{k}}\right)=\mathrm{R}_{\mathrm{j}}\left(\mathrm{DP}, \mathrm{D}_{\mathrm{k}}\right) \quad \forall \mathrm{D}_{\mathrm{k}} \in \mathrm{D}
\end{aligned}
$$

Now, substituting the values of crePoss and $R_{j}^{\prime}\left(D P, D_{k}\right)$ in relation (3), the following relation will be resulted:

$$
\begin{aligned}
& \left.\mathrm{R}_{\mathrm{F}}\left(\mathrm{DP}, \mathrm{D}_{\mathrm{k}}\right)=\min \left(\mathrm{R}_{1}^{\prime}\left(\mathrm{DP}, \mathrm{D}_{\mathrm{k}}\right), \mathrm{R}_{\mathrm{j}}\left(\mathrm{DP}, \mathrm{D}_{\mathrm{k}}\right)\right) \wedge 1\right) \\
& \forall \mathrm{DM}_{1} \in \mathrm{DM}-\left\{\mathrm{DM}_{\mathrm{j}}\right\} \\
& \mathrm{R}_{\mathrm{F}}\left(\mathrm{DP}, \mathrm{D}_{\mathrm{k}}\right)=\min \left(\mathrm{R}_{1}^{\prime}\left(\mathrm{DP}, \mathrm{D}_{\mathrm{k}}\right), \mathrm{R}_{\mathrm{j}}\left(\mathrm{DP}, \mathrm{D}_{\mathrm{k}}\right)\right) \\
& \forall \mathrm{DM}_{1} \in \mathrm{DM}-\left\{\mathrm{DM}_{\mathrm{j}}\right\}
\end{aligned}
$$

The above relation shows that only the jth agent decision, $\mathrm{Rj}(\mathrm{DP}, \mathrm{DK})$, directly affects the final decision. The impact of the other agents decisions depends on their credibility possibility values.

Property 7: Since the Max and Min operators have commutatively property then considering relation (3) it is deduced that our fusion operator also satisfies the property number 7 .

Property 8: According to relation (3), RF, is computed as a conjunction of $\min \left(\mathrm{R}_{\mathrm{i}}^{\prime}\left(\mathrm{DP}, \mathrm{D}_{\mathrm{k}}\right)\right)$ and $\max \left(\operatorname{crePoss}_{\mathrm{i}}\right)$ then rank of the final decision, $R F$, cannot exceed the maximum value of the credibility possibility of the agents.

\section{RESULTS AND DISCUSSION}

Our goal is to fuse agent responses to a query in order to improve the accuracy of answer and reduce the uncertainty. As agents have different degree of credibility in response to a query, agent responses should be fused with considering the answers credibility. In this research, we first suggest a method for assigning credibility to each agent response. This approach is based on possibility theory. According to this approach, we reach a fuzzy equation set which is solved by use of fuzzy markov chain. The assigned credibility value is of the type of possibility and have a fuzzy nature. In the next stage, we assume that in response to any query each agent produce a fuzzy answer set. Afterwards we suggest a new operator for fusing these fuzzy answer sets with considering response credibility. This operator has a set of properties which is necessary for information fusion. We discussed eight properties in this regard and then we proof that this operator has these properties. The proposed operator modifies the rank of agents' answers considering the credibility of agent which gives it and then obtains a final answer with higher degree of credibility.

\section{REFERENCES}

1. Avrachenkov, K.E. and E. Sanchez, 2002. Fuzzy markov chains and decision-making. Fuzzy Optimization and Decision Making, 1(2): 143-159.

2. Roger M. Cooke, 1991. Experts in Uncertainty. Oxford Press, Oxford, UK.

3. Dubois, D. and H. Prade, 1992. Combination of Fuzzy Information in the Framework of Possibility Theory. Data Fusion in Robotics and Machine Intelligence, 481-505. Academic Press. 
4. Pipino, L., Yang W. Lee and Y. Wang Richard, 2002. Data quality assessment. Commun. ACM., 45 (4): 211-218.

5. Chung, H. Wu, 2004. Fuzzy credibility estimation using Bayesian approach Comput. Ind. Eng., 46: 467-493.

6. Cooke, R.M., 1991. Experts in Uncertainty. Oxford Press, Oxford, UK.

7. Delmotte, F. and P. Borne, 1998. Modeling of credibility with possibility theory. IEEE Trans. Syst. Man Cybernetics, Part A., 28 (1): 78-88.

8. Guo, P., H. Tanaka and M. Inuiguchi, 2000. Selforganizing fuzzy aggregation models to rank the objects with multiple attributes. IEEE Trans. Syst. Man Cybernetics, Part A., 30 (5): 573-580.

9. Prade, H., 1985. Reasoning with fuzzy default values. Proceedings of the 5th International Symposium on Multiple-Valued Logic, Kingston, Ont., May, 191-197.
10. Weiss, G., 1999. Multiagent system. A Modern Approach to Distributed Artificial Intelligence. The MIT Press. (ISBN 0-262- 23203-0).

11. Yager Ronald, R., 2000. Approximate reasoning and conflict resolution. Int. J. Approx. Reasoning, 25 (1): 15-42.

12. Yager Ronald, R., 2004. A framework for multisource data fusion. Inf. Sci., 163 (1-3): 175-200.

13. Yager Ronald, R., 2001. Fusion of multi-agent preference orderings. Fuzzy Sets Syst., 117 (1): 1-12.

14. Yager Ronald, R., 2003. Induced aggregation operators. Fuzzy Sets Syst., 137 (1): 59-69.

15. Yager Ronald, R., 1992. On considerations of credibility of evidence. Int. J. Approx. Reasoning, 7 (1-2): 45-72.

16. Yager Ronald, R., 2004. On the determination of strength of belief for decision support under uncertainty-Part I: generating strengths of belief. Fuzzy Sets Syst., 142 (1): 117-128. 